\title{
Cost-Effectiveness of Asenapine in the Treatment of Patients with Bipolar I Disorder with Mixed Episodes in an Italian Context
}

\author{
Chiara Caresano - Guido Di Sciascio - Andrea Fagiolini - Giuseppe Maina • \\ Giulio Perugi $\cdot$ Claudio Ripellino $\cdot$ Claudio Vampini
}

To view enhanced content go to www.advancesintherapy.com

Received: June 6, 2014 / Published online: July 24, 2014

(c) The Author(s) 2014. This article is published with open access at Springerlink.com

\begin{abstract}
Introduction: Bipolar disorder is a chronic disease characterized by periods of mania or hypomania, depression, or a combination of both (mixed state). Because bipolar disorder is one of the leading causes of disability, it represents an important economic burden on society. Asenapine (ASE) is a new secondgeneration antipsychotic developed and approved for the treatment of manic or mixed episodes associated with bipolar disorder. The objective of the present study was to assess the
\end{abstract}

Electronic supplementary material The online version of this article (doi:10.1007/s12325-014-0139-3) contains supplementary material, which is available to authorized users.

C. Caresano

Department of Psychiatry, Lundbeck Italia S.p.A, Milan, Italy

G. Di Sciascio

Department of Psychiatry, Policlinico Hospital, Bari, Italy

\section{A. Fagiolini}

Department of Molecular Medicine, University of

Siena, Siena, Italy

G. Maina

Department of Neurosciences, University of Torino, Turin, Italy cost-effectiveness of ASE compared to olanzapine (OLA) in the treatment of patients experiencing mixed episodes associated with bipolar I disorder in the context of the Italian National Health Service (NHS).

Methods: A pharmacoeconomic model was developed to simulate the management of Italian bipolar I patients with mixed episodes over a 5-year time horizon by combining clinical parameters with resource utilization. An expert panel of Italian psychiatrists and health economists was responsible for adapting a UK model to the Italian context. The primary outcome measure of the economic evaluation was the incremental cost effectiveness ratio, where effectiveness is measured in terms of

G. Perugi

Department of Clinical and Experimental Medicine, University of Pisa, Pisa, Italy

G. Perugi

Institute of Behavioural Sciences "G. De Lisio", Pisa, Italy

C. Ripellino $(\bowtie)$

CSD Medical Research S.R.L., Milan, Italy

e-mail: claudio.ripellino@cegedim.com

C. Vampini

Department of Mental Health, Ospedale Civile

Maggiore, Verona, Italy 
quality adjusted life-years gained. Scenario analyses, sensitivity analyses, and a probabilistic sensitivity analysis were performed to test the robustness of the model.

Results: This pharmacoeconomic model showed that ASE resulted to be dominant over OLA; in fact, ASE was associated with lower direct costs (derived largely by the savings from hospitalizations avoided) and also generated a better quality of life. Results were robust to changes in key parameters; both scenario analyses and sensitivity analyses demonstrated model reliability.

Conclusions: Results from this study suggest that the management of bipolar I patients with mixed episodes using ASE as alternative to OLA can lead to cost saving for the Italian NHS and improve patients quality of life.

Keywords: Asenapine; Bipolar disorder; Costeffectiveness analysis (CEA); Mixed episodes; Olanzapine; Psychiatry

\section{INTRODUCTION}

Bipolar disorder is a chronic disease characterized by periods of mania or hypomania (episodes of elevated moods, extreme irritability, decreased sleep, and increased energy), depression (overwhelming feelings of sadness, anhedonia, suicidal thoughts) or a combination of both (named as mixed state). The exact cause of the condition is unknown, but genetic, physiological, neurological, psychosocial, and environmental factors may be involved. According to the Diagnostic and Statistical Manual of Mental Disorders, Fourth Edition, the diagnosis of bipolar type I requires the presence or the history of at least one manic or mixed episode, whereas bipolar disorder type II differs from type I only by the presence of hypomania but no manic episodes [1].

The prevalence of bipolar I disorder in Italy is estimated to be approximately $1-2 \%$, even if the true prevalence is likely to be much higher because it is frequently under-diagnosed, largely due to the fact that it is difficult to diagnose correctly [2]. Because bipolar disorder is one of the leading causes of disability, especially in active populations, it represents an important economic burden on society [3]. A study evaluating the cost of bipolar disorder in the UK has estimated a cost of $£ 198.7$ million per year, with hospitalization accounting for the largest component of direct costs [4].

Mixed episodes are a common feature of bipolar I disorder and they are associated with more severe symptoms and outcomes $[5,6]$; moreover, this type of episode tends to last longer than manic and depressive episodes [7] and is more commonly associated with substance abuse, anxiety disorders, and suicidality [8-10]. Treatment of bipolar mixed states is often challenging as response is usually poorer than in manic or depressive episodes [11] and involves the use of lithium (Li), sodium valproate (VPA), and second-generation antipsychotics (SGAs) [12]. Currently, the best evidence for efficacy as monotherapy for the acute treatment of mixed state is provided for anticonvulsants followed by the atypical antipsychotics: aripripazole, asenapine (ASE), paliperidone, and ziprasidone [13]. There was also very strong evidence for the use of olanzapine (OLA) as monotherapy or as addon to Li or VPA [13].

In 2013, the first meta-analysis of the efficacy of SGAs in mixed episodes was published, suggesting that some of these drugs are effective in reducing not only manic but also depressive symptoms in patients with mixed episodes [14]. SGAs, particularly ASE, OLA, 
aripriprazole, paliperidone, risperidone, and ziprasidone were reported to be efficacious on maniac symptoms of mixed episodes, while ASE and OLA also appear to be moderately efficacious in treating depressive symptoms of mixed episodes $[14,15]$.

ASE is a new SGA developed and approved in the USA for the acute treatment, as monotherapy or adjunctive therapy with either Li or VPA, of manic or mixed episodes associated with bipolar I disorder. Whether used as monotherapy or as adjunctive therapy with $\mathrm{Li}$ or VPA, the use of ASE in responding patients is generally recommended by the Food and Drug Administration to be continued beyond the acute response [16]. In Europe, in September 2010, the European Medicines Agency has authorized the marketing of this molecule for the treatment of moderate to severe manic episodes associated with bipolar I disorder in adults.

Focusing on mixed episode patients, Azorin et al. [17] assessed the efficacy of ASE using post hoc analyses on pooled data from two identical 3-week, randomized, double-blind, placeboand OLA-controlled trials $[18,19]$ and their 9-week double-blind, OLA-controlled extension study [20]. At week three, ASE was found to be significantly superior to both placebo and OLA both on manic and depressive symptoms improving Montgomery-Åsberg Depression Rating Scale (MADRS) and Young Mania Rating Scale (YMRS) scores. At week 12, further decrease in YMRS and in MADRS total scores was observed with ASE, although not reaching a statistically significant difference. Although no statistically significant difference was observed between ASE and OLA in composite response rate or mean reduction on either score, these findings suggest a potential difference in the way the two drugs work in mixed episodes.
Even though cost-effectiveness of different SGAs in the treatment of patients experiencing a manic episode associated with bipolar I disorder has been studied in numerous analyses and in different contexts [21-26], only a few studies $[27,28]$ have explored the role of these drugs in patients with mixed episodes.

The current study evaluates from an Italian National Health Service (NHS) perspective, the cost-effectiveness of ASE compared to OLA in the treatment of patients experiencing mixed episodes associated with bipolar I disorder. Comparison against SGAs was only possible with OLA due to lack of randomized studies comparing efficacy of ASE versus other antipsychotics in the treatment of the subpopulation with mixed episodes. Furthermore, OLA was the best comparator based on proven efficacy and market-share [13].

\section{METHODS}

The analysis in this article is based on previously conducted studies and does not involve any new studies of human or animal subjects performed by any of the authors.

\section{Model Introduction}

The pharmacoeconomic model was developed to simulate the management of Italian bipolar I patients with mixed episodes over a five-year time horizon by combining clinical parameters with resource utilization.

An expert panel, composed of five psychiatrists from different Italian regions and two health economists from an independent agency, was responsible for adapting a model developed by Sawyer et al. [27] from the UK into an Italian perspective. 
Efficacy was informed by a post hoc analysis of two short-term clinical trials, with response measured as a composite MADRS and YMRS [17]. Probabilities of discontinuation and relapse to manic, mixed, and depressive episodes were sourced from published meta-analyses [29, 30].

The primary outcome measure of the economic evaluation is the incremental costeffectiveness ratio (ICER), where effectiveness is measured in terms of quality-adjusted life years (QALYs) gained.

Because costs were considered from the Italian NHS perspective, only direct medical costs, expressed in Euro, could be evaluated. The pharmacoeconomic model was developed using Microsoft Office Excel 2007 (Microsoft Corporation, Albuquerque, NM, USA) software.

\section{Description of Model}

The model comprises an acute phase and a maintenance phase. The acute phase (Fig. 1) is designed using a standard decision tree model, and it covers the three three-week initial treatment periods. The maintenance phase is designed using a Markov model, and it follows patients for four-week cycles for a five-year followup period as their condition stabilizes and the goal of treatment shift into relapse prevention. A survey on treatment guidelines [31] demonstrates a lack of consensus regarding the appropriate time to change treatment strategies in case of partial or non-response during an acute manic episode, with recommendations ranging from 2 to 12 weeks. In the absence of firm evidence and given the high risk of suicide in mixed episodes, a shorter duration (3 weeks) during which to assess response to treatment was considered appropriate.

Hypothetical patients enter the acute phase of the model experiencing a mixed episode being allocated to either ASE or OLA treatment arms. Non-responder patients will have an adjunctive therapy with VPA (ASE/OLA + VPA; 2nd line), and, if they are still not responding for the 2nd line, a switch to adjunctive Li (ASE/ OLA $+\mathrm{Li}$; 3rd line). The sequence of adjunctive therapy with VPA as 2nd line and Li as third line was based on the fact that most guidelines mention the superiority of VPA over Li to treat mixed episodes [13]. These assumptions were informed by clinical expert opinion.

After the 9-week model acute phase, patients non-respondent to any of the treatment lines enter the Markov model as "Non-responder" on triple therapy (ASE/OLA + VPA + Li). If the response criteria are met for any treatment lines, patients enter directly in the

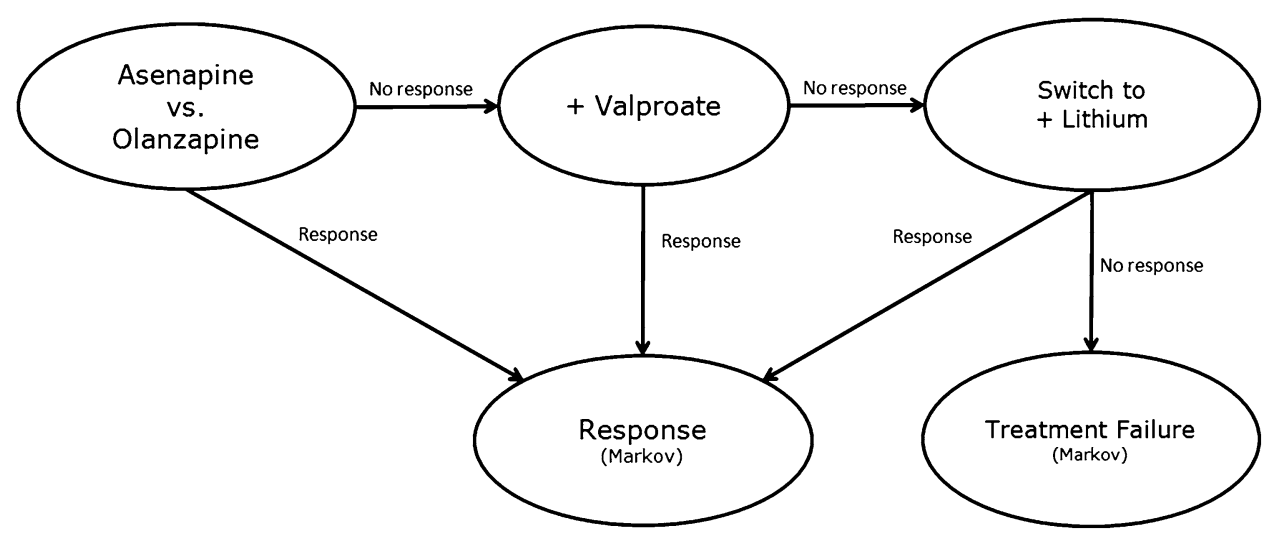

Fig. 1 Acute phase model structure 


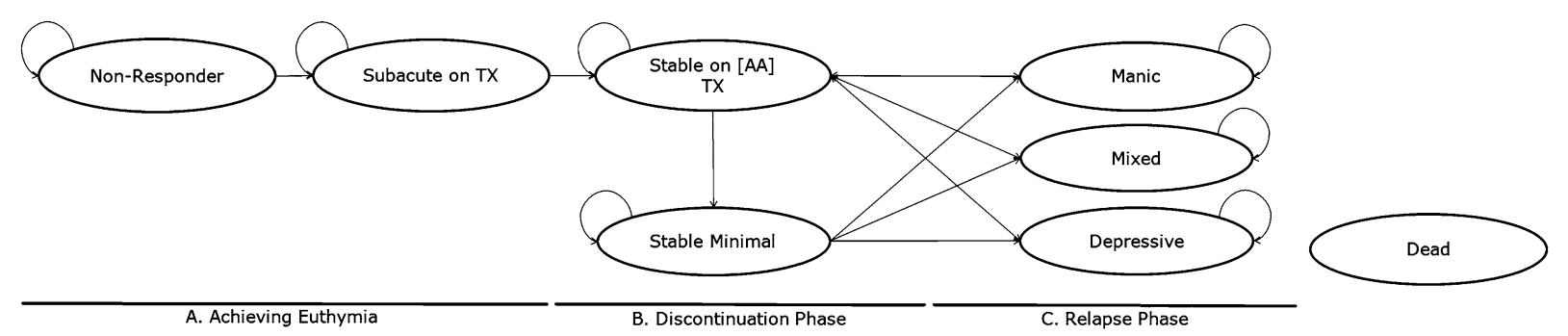

Fig. 2 Maintenance phase model structure. $T X$ treatment, $A A$ atypical antipsychotic

maintenance Markov model in the "Subacute on Tx" health state.

The maintenance phase of the economic model (Fig. 2) is designed to follow hypothetical patients for 5 years after the index mixed episode, irrespective of whether they did or did not respond to treatment during the acute phase. Five years was deemed a sufficient length of time by clinical experts to capture the longerterm aspects of bipolar disorder treatment, such as stabilization after an acute episode, possible discontinuation of therapy, management and prevention of relapse episodes, and mortality.

As stated above, patients who have responded to either monotherapy or adjunctive treatments (1st-, 2nd-, or 3rd-line responders) in the acute phase enter the maintenance phase of the economic model in a subacute health state (i.e., a transitory state in which all responding patients must pass before reach stability), whereas patients who have not responded to any therapy in the acute phase will enter the maintenance phase as non-responders.

Patients will stay in these health states until they respond to treatment (if non-responders, transition from "Non-responder" state to "Subacute on Tx" state) and achieve euthymia (transition from "Subacute on $\mathrm{Tx}$ " to "Stable on atypical antipsychotic [AA] Tx"). Once patients achieve euthymia while on treatment, they may discontinue treatment with SGA remaining with VPA alone or Li alone (defined minimal treatment and represented in the model as
"Stable minimal $T x$ " state). Stable patients (either on regular antipsychotic treatment or on the minimal treatment described above) can relapse and experience a manic, mixed, or depressive episode. Following relapse, patients across both arms switch to treatment with quetiapine (QTP) + VPA or QTP + Li (a 50/50 split is assumed), remaining on this treatment until the end of the modeled time horizon. This simplified approach ensures that all patients follow the same long-term prognosis and costs. At any point in the model, patients can die.

\section{Model Parameters}

\section{Response, Relapse, Discontinuation, and other Probabilities}

Response to the acute phase with first line monotherapy (ASE or OLA) is defined in this model as $\geq 50 \%$ improvement from baseline in the YMRS and MADRS scores within 3 weeks, and sourced from the post hoc analysis of the ARES trial on mixed patients [17].

Comparative response rates for ASE and OLA in combination with VPA or $\mathrm{Li}$ on the composite YMRS and MADRS outcome are not available from clinical trial data; therefore, for the adjunctive therapies (2nd and 3rd line treatments), differently from the UK model in which 95\% confidence intervals upper limits were used, the authors applied the ASE + VPA/ $\mathrm{Li}$ response data from Apollo 12 (Clinicaltrials.gov \#NCT00145470) trial 
assuming the same response for both ASE and OLA (Table 1) [32]. This assumption was deemed reasonable by clinical expert opinion.

The annual risks of relapse used in the maintenance phase model for each treatment arm are reported in Table 1. Most of these values were sourced from a systematic review and network meta-analysis by Soares-Weiser et al. [30]. As ASE was not available in this review, it was assumed that the annual risk of relapse for ASE was the same as for OLA. Similarly, as no data were available on the efficacy of combination therapies in relapse prevention, it was assumed that risk of relapse was related to the last added treatment.

Since probabilities of relapse from SoaresWeiser et al. [30] were stratified by manic/mixed or depressive episode, the model needed a further breakdown between patients experiencing a recurrent manic episode versus a recurrent mixed episode. For this reason, the authors applied the probability of a relapse split between mania and mixed episodes from the study by Vieta and colleagues (53.3\% mania and $46.7 \%$ mixed) [33].

Treatment discontinuation in the model is defined as patients discontinuing their antipsychotic treatment and moving on to a "minimal treatment", which was assumed to be VPA alone for $50 \%$ of patients and $\mathrm{Li}$ alone for the other $50 \%$. As in the UK model, probability of discontinuing treatment with the initial drug during periods of disease stability was calculated by applying a risk ratio from a published metaanalysis of clinical trials [34] to an underlying baseline probability of discontinuing on placebo. The placebo discontinuation rates are based on a published discrete-event simulation model for patients with acute bipolar depression [29]. Again, as ASE-treatment discontinuation risk was not available in the meta-analysis, it was assumed that the treatment discontinuation probability for ASE was the same as for OLA (Table 1).

Patients who failed to respond to the 1st-, 2nd-, and 3rd-line treatments in the acute phase enter the maintenance phase as non-responders and on triple therapy (AA + VPA + Li). Due to lack of data, the authors have assumed a $35 \%$ per cycle probability of reaching the subacute health state and varied the base case probability in a sensitivity analysis from $0 \%$ to $100 \%$.

The subacute health state was implemented in the model as a temporary tunnel state, through which responding patients must transition before achieving stable euthymia. From this temporary tunnel state, a monthly probability of achieving euthymia was estimated to be $60 \%$ based on expert opinion. Also this probability has been tested by the authors with a sensitivity analysis from $0 \%$ to $100 \%$.

The mortality risk, stratified by causes (suicide and non-suicide), was calculated starting from the interim life tables for Italy [35] and applying the general population annual risk of suicide from the same source. Finally, the authors applied standardized mortality ratios for suicide (11.53) and nonsuicide (1.69) retrieved from Angst et al. [36] to define the mortality risk among patients with bipolar I disorder.

\section{Adverse Event}

The model incorporates two common adverse events due to antipsychotic medication: weight gain and extrapyramidal symptoms (EPS), whose incidences, that are different according to drug regimen, were retrieved from several trials [17, 33, 37, 38]. Weight gain is associated with all bipolar disorder drugs; so, this adverse event was associated with all drugs. For combination therapies, the incidence of weight gain is set to equal that of the drug 
Table 1 Response rates, annual risks of relapse, treatment discontinuation probabilities, and incidence values for weight gain and EPS

\begin{tabular}{|c|c|c|c|}
\hline Treatment drug & \multicolumn{2}{|c|}{ Response (95\% CI) } & Source \\
\hline \multicolumn{4}{|l|}{ Response rates } \\
\hline ASE & \multicolumn{2}{|c|}{$0.463(0.340 ; 0.589)$} & {$[17]$} \\
\hline OLA & \multicolumn{2}{|c|}{$0.375(0.274 ; 0.485)$} & {$[17]$} \\
\hline $\mathrm{ASE}+\mathrm{VPA} / \mathrm{Li}$ & \multicolumn{2}{|c|}{$0.342(0.268 ; 0.416)$} & {$[32]$} \\
\hline $\mathrm{OLA}+\mathrm{VPA} / \mathrm{Li}$ & \multicolumn{2}{|c|}{$0.342(0.268 ; 0.416)$} & Assumed the same as ASE $+\mathrm{VPA} / \mathrm{Li}$ \\
\hline Treatment drug & Manic/Mixed & Depression & Source \\
\hline \multicolumn{4}{|c|}{ Annual risks of relapse } \\
\hline ASE & 0.08 & 0.14 & Assumed the same as OLA \\
\hline OLA & 0.08 & 0.14 & {$[30]$} \\
\hline VPA & 0.23 & 0.05 & {$[30]$} \\
\hline $\mathrm{Li}$ & 0.20 & 0.07 & {$[30]$} \\
\hline $\mathrm{Li}+\mathrm{VPA}$ & 0.23 & 0.05 & Assumed to be the max value between VPA and $\mathrm{Li}$ \\
\hline $\mathrm{QTP}+\mathrm{Li} / \mathrm{VPA}$ & 0.14 & 0.22 & {$[33]$} \\
\hline Minimal TX & 0.215 & 0.06 & Average of $\mathrm{Li}$ and VPA \\
\hline $\begin{array}{l}\text { Treatment drug } \\
\text { Tru }\end{array}$ & \multicolumn{2}{|c|}{ Probability } & Source \\
\hline
\end{tabular}

Treatment discontinuation probabilities

$\begin{array}{ll}\text { Placebo } & 0.017 \\ \text { ASE } & 0.013 \\ \text { OLA } & 0.013 \\ \mathrm{VPA} & 0.014 \\ \mathrm{Li} & 0.016 \\ \mathrm{Li}+\mathrm{VPA} & 0.014 \\ \text { QTP }+\mathrm{Li} / \mathrm{VPA} & 0.012\end{array}$

[34]

Assumed the same as OLA

[29]

[29]

[29]

Assumed to be the max value between VPA and Li [29]

\begin{tabular}{llll}
\hline Treatment drug & Weight gain & EPS & Source \\
\hline Incidence values for weight gain and EPS & & & \\
ASE & 0.138 & 0.112 & {$[17]$} \\
OLA & 0.0764 & 0.098 & {$[17]$} \\
VPA & 0.079 & 0.032 & {$[37]$} \\
Li & 0.029 & 0.028 & {$[38]$} \\
QTP + VPA + Li & 0.144 & 0.0092 & {$[33]$} \\
\hline
\end{tabular}

$A S E$ asenapine, EPS extrapyramidal symptoms, $L i$ lithium, $O L A$ olanzapine, $Q T P$ quetiapine, $V P A$ sodium valproate 
with the highest incidence. EPS is typically associated with atypical antipsychotics and not with mood stabilizers; so, EPS are assumed to occur only during the atypical antipsychotics treatment. Both adverse events are assumed to occur independently. The incidence values for weight gain and EPS are reported in Table 1.

\section{Utilities}

Utility values for the model health states and adverse events were derived from the UK model [24], except for inpatient and outpatient mixed episodes utilities; since no data were available in literature, the authors agreed to set the same values as those set for mania (Table 2).

The utility values of the general population applied in the model were converted to multipliers using the formulae of Ara and Brazier [39], which take into account the ageand gender-distribution relative to the study population.

Table 2 Utility values

\begin{tabular}{|c|c|c|}
\hline Condition & Utility value & Source \\
\hline Subacute & 0.8 & [39] \\
\hline Stable & 0.84 & {$[17]$} \\
\hline Mania IP & 0.245 & [39] \\
\hline Mania OP & 0.585 & [39] \\
\hline Mixed IP & 0.245 & $\begin{array}{l}\text { Assumed the same as } \\
\text { Mania IP }\end{array}$ \\
\hline Mixed OP & 0.585 & $\begin{array}{l}\text { Assumed the same as } \\
\text { Mania OP }\end{array}$ \\
\hline Depression IP & 0.29 & [39] \\
\hline Depression OP & 0.605 & {$[46]$} \\
\hline Weight gain & 0.825 & {$[47]$} \\
\hline EPS & 0.722 & {$[47]$} \\
\hline
\end{tabular}

In order to derive the condition-specific utility values for the model health states and adverse events, a multiplicative approach was used considering multipliers defined according to different health states and adverse events.

\section{Costs and Resource Utilization}

This model considered only direct medical costs including those associated with drug acquisition, general practitioner (GP) visits and specialist visits, examinations, and hospitalizations. This model is set in the Italian NHS perspective. Therefore, only the costs directly reimbursed by the NHS were considered. Since a 5-year time horizon was set, a discounting of $0.035 \%$ was applied.

Drug costs were calculated based on prices listed on the Italian Drug Agency website [40]. For generic drugs, the authors considered prices reimbursed by the NHS, whereas for ASE, the only branded drug, the authors considered the public price applying a discount for NHS (12.5\% for drugs between $€ 103.29$ and $€ 154.94)$ after VAT. Treatment doses used in the analysis were the weighted average of the daily doses found in literature. The unit costs per $\mathrm{mg}$, daily doses (mg), and daily costs for the drugs used in this model are reported in Table 3.

In order to test the dosages applied in the model, the authors developed a scenario analysis adopting the recommended daily doses from the summary of product characteristics (SPCs) for both the acute phase and the maintenance phase (Table 3).

Resource utilization was differentiated according to drug regimen and for the acute and maintenance phases, and it was assessed to reflect the Italian standard clinical practice.

Full blood count, liver panel, blood urea, creatinine, thyroid function (i.e., thyroidstimulating hormone), serum Li 
Table 3 Treatment costs considering daily doses from literature and summary of product characteristics (SPCs)

\begin{tabular}{|c|c|c|c|c|c|}
\hline Treatment & \multicolumn{2}{|c|}{ Unit cost per mg $(€)$} & \multicolumn{2}{|c|}{ Daily dose (mg) } & Daily cost $(€)$ \\
\hline \multicolumn{6}{|c|}{ Treatment costs considering daily doses from literature } \\
\hline Asenapine & \multicolumn{2}{|l|}{0.22} & \multicolumn{2}{|l|}{18.3} & 4.07 \\
\hline Olanzapine & \multicolumn{2}{|l|}{0.16} & \multicolumn{2}{|l|}{14.8} & 2.39 \\
\hline Quietiapine & \multicolumn{2}{|l|}{0.003} & \multicolumn{2}{|l|}{613.8} & 2.05 \\
\hline Valproate & \multicolumn{2}{|l|}{0.00004} & \multicolumn{2}{|l|}{$1,735.4$} & 0.68 \\
\hline Lithium & \multicolumn{2}{|l|}{0.00002} & \multicolumn{2}{|l|}{$1,126.3$} & 0.19 \\
\hline Lithium + Valproate & \multicolumn{2}{|l|}{ - } & \multicolumn{2}{|l|}{-} & 0.87 \\
\hline \multirow[t]{2}{*}{ Treatment } & \multicolumn{2}{|c|}{ Daily dose (mg) } & & \multicolumn{2}{|c|}{ Daily cost $(€)$} \\
\hline & Acute & Maintenance & & Acute & Maintenance \\
\hline \multicolumn{6}{|c|}{ Treatment costs considering daily doses from SPCs } \\
\hline Asenapine & 20 & 10 & & 4.45 & 2.22 \\
\hline Olanzapine & 15 & 10 & & 2.41 & 1.61 \\
\hline Quietiapine & - & 600 & & - & 2.00 \\
\hline Valproate & 1,500 & 1,500 & & 0.59 & 0.59 \\
\hline Lithium & 1,200 & 1,200 & & 0.20 & 0.20 \\
\hline Lithium + Valproate & - & - & & 0.79 & 0.79 \\
\hline
\end{tabular}

concentration, electrocardiogram, electrolytes, glucose test, lipid profile, prolactin, serum valproic acid concentration, amylase, and ammonium were considered and their costs were taken from the 2014 pricelist from Italian Ministry of Health website (Tariffa Unica Convenzionale [TUC]) [41].

Health state associated costs were estimated to differ on a yearly base and depending upon the episode type (i.e., acute manic, mixed, and depressive episode) and health state. The assumptions of health state costs follow the ones described by Soares-Weiser et al. [30] and adopted also in the UK model [27]. Since the Soares-Weiser approach classified services based on the type of management (inpatient, outpatient, and crisis resolution team), the authors readapted this approach to the Italian context (Table 4) introducing Day Hospital service. Furthermore, an adjunctive scenario analysis using a different management breakdown discussed by the expert panel was performed (Table 4). Since Soares-Weiser et al. [30] reported information on mania and depression only, the authors assumed the same management breakdown and healthcare services utilization for mixed episode and mania.

Healthcare services costs and utilizations are presented in Table 4 and unit costs were retrieved from the TUC 2014. The authors assumed that the cost for the stable health state is the baseline cost and any relapse event represents an additional cost.

As mentioned before, the main adverse events for patients on treatment for bipolar I disorder are weight gain and EPS. The weight gain costs for the acute phase were a dietician 


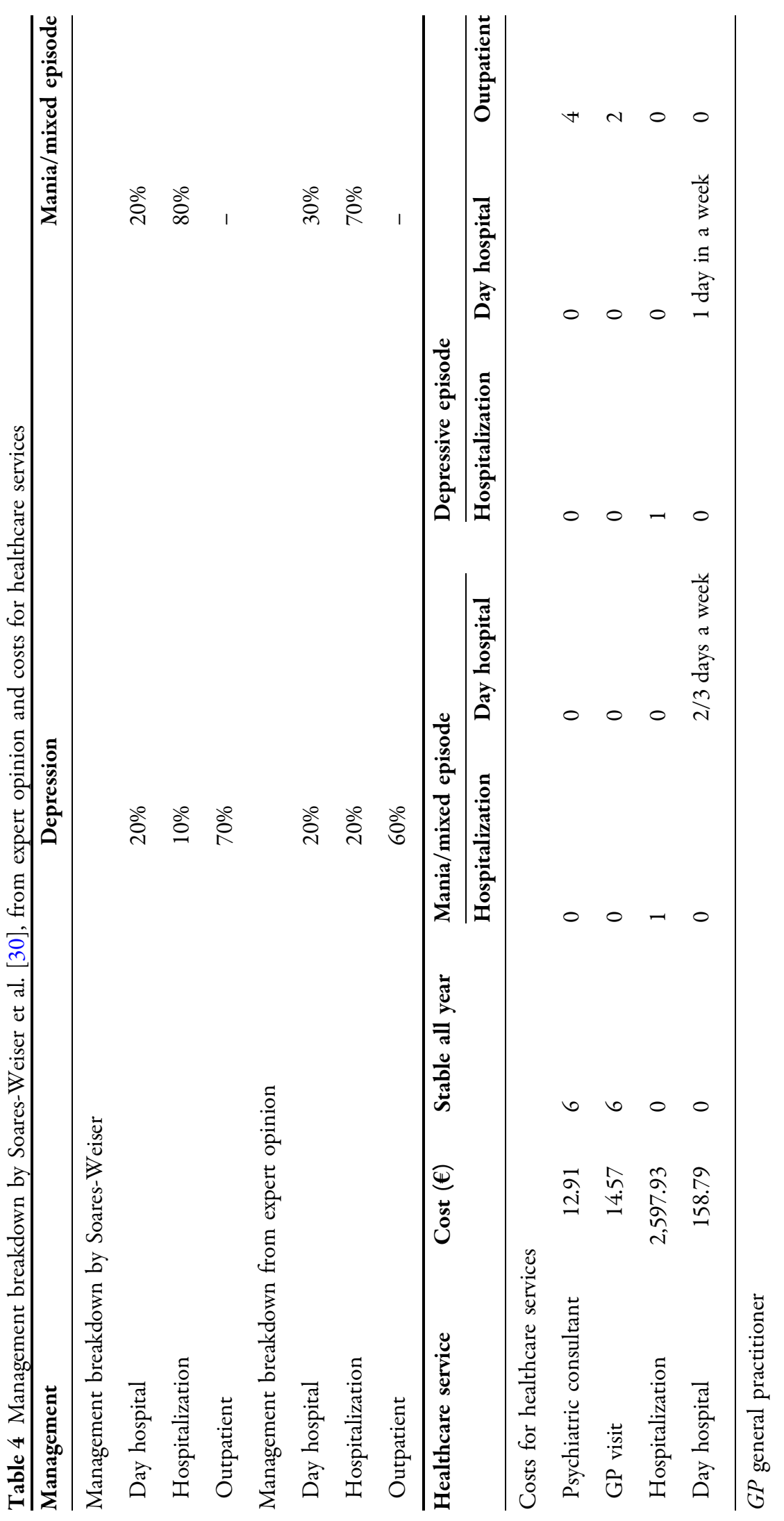


visit cost and an additional GP visit cost; no cost was associated to weight gain in the maintenance phase. For EPS only medication costs (biperidene $6 \mathrm{mg}$ daily for 3 months) were considered, both in acute and maintenance phases.

\section{Cost-Effectiveness and Sensitivity Analyses}

Cost-effectiveness analysis (CEA) is a method for assessing the health gains in relation with the costs of the different health interventions.

The main outcomes of the model are mean costs and QALYs for treatment strategies and the ICERs, which are the observed differences in costs divided by differences in outcomes between two alternative programs. The ICER should be interpreted as the additional cost required to gain an additional unit of health outcome (i.e., QALY) when providing one treatment rather than another one.

The associated probabilities, as long as treatment costs, are subject to uncertainty, so it may be necessary to test robustness of the results obtained changing the model inputs or using alternative parameters. In order to test the robustness of the model results, sensitivity analyses, scenario analyses, and probabilistic analysis were performed.

Based on input from the expert panel, the following analyses were developed:

- Scenario analysis on different drugs doses;

- Scenario analysis on different episode management breakdown;

- Sensitivity analysis on transition probability from non-responder to subacute state;

- Sensitivity analysis on transition probability from subacute state to stable state.

Furthermore, a probabilistic sensitivity analysis (PSA), in which all model parameters have been varied simultaneously, was also performed.

\section{RESULTS}

\section{Base Case Analysis}

Table 5 presents the results of the base case costeffectiveness analysis. ASE generated an increase in QALYs of 0.0240 at average lower total costs (i.e., €461.3 less) versus OLA, dominating its comparator. Although the incremental treatment costs $(€ 1,134.5)$ are greater for ASE than for OLA, savings in health state costs generated by avoiding hospitalization $(€ 1,588.8)$ offset the total difference (Table 5$)$.

An incremental gain of 0.0106 QALYs is observed when the impact of adverse events is excluded from quality of life calculations. Also on this outcome, ASE dominates OLA in our cost-effectiveness model (Table 5).

When only the acute phase is considered (Table 5), referring to the first 9 weeks of treatment after the mixed episode, ASE generates greater benefits than OLA $(0.0045$ more QALYs and 0.0041 more QALYs when the impact of adverse events is excluded) at lower costs $(€ 1,065.4$ less). This result indicates that the incremental QALYs and savings are derived largely from the earlier response to treatment during the acute phase.

\section{Scenario Analyses}

Table 6 describes the results of the scenario analysis on different drug doses in which the average doses derived from clinical studies used in the base case were changed to the daily doses from the SPCs (see Table 3).

Compared to OLA, also here ASE dominates OLA, with a lower incremental total cost 
Table 5 Base case results

\begin{tabular}{|c|c|c|c|c|c|c|}
\hline Strategy & $\begin{array}{l}\text { QALYs } \\
\text { w/o AEs }\end{array}$ & $\begin{array}{l}\text { QALYs } \\
\text { w/AEs }\end{array}$ & $\begin{array}{l}\text { Treatment } \\
\text { costs }(€)\end{array}$ & AE costs $(€)$ & $\begin{array}{l}\text { Health state } \\
\text { costs }(€)\end{array}$ & $\begin{array}{l}\text { Total } \\
\text { costs }(€)\end{array}$ \\
\hline \multicolumn{7}{|l|}{ Base case results } \\
\hline ASE strategy & 3.6826 & 3.6495 & $4,716.2$ & 86.8 & $28,107.9$ & $32,911.0$ \\
\hline OLA strategy & 3.6720 & 3.6256 & $3,581.7$ & 93.9 & $29,696.7$ & $33,372.3$ \\
\hline Incremental & 0.0106 & 0.0240 & $1,134.5$ & -7.1 & $-1,588.8$ & -461.3 \\
\hline \multicolumn{7}{|c|}{ Base case results considering only the acute phase ( 9 weeks) } \\
\hline ASE strategy & 0.0859 & 0.0833 & 119.2 & 19.4 & $18,471.7$ & $18,610.3$ \\
\hline OLA strategy & 0.0818 & 0.0788 & 85.1 & 31.6 & $19,559.1$ & $19,675.7$ \\
\hline Incremental & 0.0041 & 0.0045 & 34.1 & -12.2 & $-1,087.4$ & $-1,065.4$ \\
\hline
\end{tabular}

AEs adverse events, ASE asenapine, OLA olanzapine, QALYs quality-adjusted life years, $w /$ with, $w / o$ without

Table 6 Scenario analyses

\begin{tabular}{lllllll}
\hline Strategy & $\begin{array}{l}\text { QALYs } \\
\text { w/o AEs }\end{array}$ & $\begin{array}{l}\text { QALYs } \\
\text { w/AEs }\end{array}$ & $\begin{array}{l}\text { Treatment } \\
\text { costs }(\boldsymbol{\epsilon})\end{array}$ & AE costs $(€)$ & $\begin{array}{l}\text { Health state } \\
\text { costs }(€)\end{array}$ & $\begin{array}{l}\text { Total } \\
\text { costs }(€)\end{array}$ \\
\hline Scenario analysis on different drugs doses & & & & \\
ASE strategy & 3.6826 & 3.6495 & $3,404.7$ & 86.8 & $28,107.9$ & $31,599.4$ \\
OLA strategy & 3.6720 & 3.6256 & $2,982.5$ & 93.9 & $29,696.7$ & $32,773.1$ \\
Incremental & 0.0106 & 0.0240 & 422.2 & -7.1 & $-1,588.8$ & $-1,173.7$
\end{tabular}

Scenario analysis on different episode management breakdown

\begin{tabular}{lllllll} 
ASE strategy & 3.6884 & 3.6553 & $4,710.7$ & 86.8 & $26,422.2$ & $31,219.7$ \\
OLA strategy & 3.6788 & 3.6322 & $3,577.0$ & 93.9 & $27,857.5$ & $31,528.4$ \\
Incremental & 0.0096 & 0.0231 & $1,133.7$ & -7.1 & $-1,435.3$ & -308.6 \\
\hline
\end{tabular}

$A E s$ adverse events, $A S E$ asenapine, OLA olanzapine, QALYs quality-adjusted life years, $w /$ with, $w / o$ without

$(€ 1,173.7$ less than OLA), with respect to the base case analysis (€461.3 less than OLA) thanks to savings in the treatment costs (€422.2 vs. $€ 1,134.5$, respectively).

The results of the scenario analysis on the episodes management breakdown as derived from the experts' opinions rather than the one used in the base case analysis (see Table 4) are described in Table 6. Costs generated by ASE and OLA are slightly lower than the ones of the base case analysis (€31,219.7 and €31,528.4, respectively) due to savings in health state costs. The consistency of results between the base case and the two scenario analyses supports the robustness of the model.

\section{Sensitivity Analyses}

A sensitivity analysis on the probability of changing health state from non-responders to subacute health state during the maintenance phase was performed. In the base case analysis, this probability was set at 35\% per cycle, while the sensitivity analyses used a probability of $0 \%$ and 100\%. Results appeared sensitive to variations in this parameter, but ASE remained 
Table 7 Sensitivity analyses

\begin{tabular}{lcc}
\hline Strategy & QALYs w/AEs & Total costs $(\boldsymbol{\epsilon})$ \\
\hline 0\% probability of reaching the subacute health state from the non-responders state & \\
ASE strategy & 3.1622 & $62,597.7$ \\
OLA strategy & 3.0619 & $68,172.8$ \\
Incremental & 0.1003 & $-5,575.1$ \\
100\% probability of reaching the subacute health state from the non-responders state & \\
ASE strategy & 3.6660 & $31,887.2$ \\
OLA strategy & 3.6447 & $32,178.1$ \\
Incremental & 0.0213 & -290.9 \\
0\% probability of reaching the stable health state from the subacute health state & \\
ASE strategy & 3.6489 & $34,039.6$ \\
OLA strategy & 3.6245 & $34,671.2$ \\
Incremental & 0.0244 & -631.6 \\
100\% probability of reaching the stable health state from the subacute health state & \\
ASE strategy & 3.6498 & $32,409.8$ \\
OLA strategy & 3.6260 & $32,795.5$ \\
Incremental & 0.0238 & -385.7 \\
\hline
\end{tabular}

$A E s$ adverse events, $A S E$ asenapine, $O L A$ olanzapine, $Q A L Y s$ quality-adjusted life years, $w /$ with

dominant over OLA throughout the entire range tested by the sensitivity analysis (Table 7).

Another sensitivity analysis was performed on the probability of reaching the stable health state from the subacute health state. In this analysis the probability was changed from the $60 \%$ in the base case analysis to a range from $0 \%$ to $100 \%$. Also in this case ASE remained dominant over OLA, similarly to the results obtained in the base case analysis (Table 7).

\section{Probabilistic Sensitivity Analyses}

In the PSA, the probabilities of response, relapse, discontinuation, and death, the health state utility values and the unit costs were probabilistically sampled 1,000 times generating 1,000 samples.
Figure 3 represents the cost-effectiveness plane in which the 1,000 estimates of mean costs and mean effectiveness resulted from the samples are plotted. In the majority of samples, treatment with ASE resulted to be associated with lower costs and increased QALYs compared to OLA (lower-right quadrant).

The higher-right quadrant represents samples that should need money investment in order to achieve additional QALYs by using ASE. In this study, due to the lack of official willingness to pay threshold in Italy, we decided to use an ICER threshold of $€ 30,000$ per QALY, slightly lower than the one recognized by the National Institute for Health and Care Excellence (NICE; £20,000-30,000) [42].

Being so, with a willingness-to-pay threshold set to $€ 30,000$ per QALY, treatment of patients 


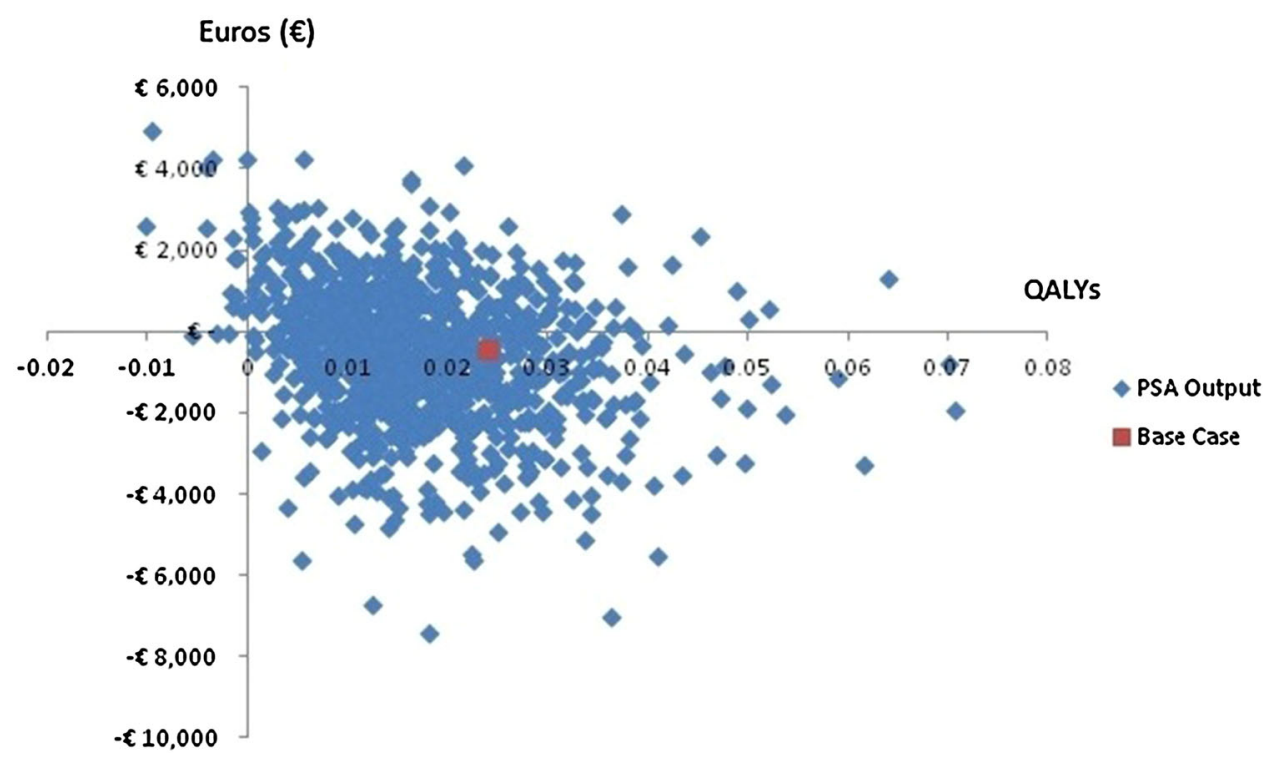

Fig. 3 PSA Scatter plot on cost-effectiveness plane for treatment with ASE versus OLA. ASE asenapine, OLA olanzapine, $P S A$ probabilistic sensitivity analysis, QALYs quality-adjusted life years

experiencing a mixed episode with ASE has a probability of $72.4 \%$ to be cost-effective compared to OLA. PSA results also demonstrate that ASE is dominant in $63.1 \%$ of simulations.

\section{DISCUSSION}

In our pharmacoeconomic model, developed to simulate the management of bipolar I patients with mixed episodes over a 5-year time period from the Italian NHS perspective, ASE results superior to OLA. In fact, ASE is associated with lower direct costs ( $-€ 461.3)$, derived largely by the savings from hospitalizations avoided $(-€ 1,588.8)$, and also generates a better quality of life $(+0.0240$ QALYs). In line with results obtained in clinical trials $[18,20]$, this pharmacoeconomic study shows that the greater health benefits and cost savings were driven by earlier response to ASE treatment during the acute phase and were well maintained during long-term follow-up.
Results were robust to changes in key parameters; both scenario analyses modeling different health status transition probabilities, drug doses, and episode management breakdown, and sensitivity analyses demonstrated model reliability, being these scenario cases results comparable to base case analysis. In particular, $63.1 \%$ out of the 1,000 PSA simulations found that ASE dominated OLA and $72.4 \%$ showed an ICER lower than $€ 30,000$.

Hospitalizations generate the most important costs, which account for about 85-90\% of total expenses and, as highlighted by Brunelle et al. [43], this burden increases because inpatients that experienced a bipolar I mixed episode may have a prolonged hospitalization.

Results of the original model developed by Sawyer et al. [27] are slightly different, with ASE not dominating OLA, but generating an ICER value $(£ 1,302)$ lower than the one usually applied by NICE [42]. Different results are mostly attributable to the different inpatient 
and outpatient management between the two Countries, as already described in the method section.

A recent cost-effectiveness analysis performed by Lachaine et al. [28] in Canada, which focused on metabolic changes and their complications on quality of life and survival, showed a dominance of ASE in comparison with OLA in both NHS and societal perspectives, with an increase of 84.84 QALYs and a cost decrease of 3,847,300 Canadian dollars (for NHS perspective, while for societal perspective is $3,878,343$ Canadian dollars) per 1,000 patients.

The foremost strength of this study is the analysis methodology itself, since CEA accounts not only for effectiveness and costs, but includes also information on relapses, hospitalizations, and adverse events. The role of pharmacoeconomic models is central when it is necessary to establish priorities in the allocation of resources in a specific therapeutic area. Indeed, they supply decision makers within healthcare systems with useful tools to make more rational and effective decisions.

The main study limitation concerns the lack of publications regarding ASE treatment of bipolar I type with mixed episode patients, and adequate comparative data for other SGAs in acute phase. In previous meta-analysis [14, 44 ] only post hoc analyses on pooled data from clinical trials by McIntyre [18-20] were found comparing ASE and OLA in mixed episodes. Therefore, the authors of this pharmacoeconomic study, similarly to the authors of the previous pharmacoeconomic study performed in the UK [27], used the post hoc analyses by Azorin et al. [17] to derive the efficacy and safety of treatments. The model was validated by expert opinions.

Whilst important basic values, such as efficacy and safety of OLA and ASE, were available from these post hoc analyses, evidence for other important parameters, i.e. efficacy of adjunctive therapies, longer-term efficacy of SGAs in relapse prevention, was not available. Therefore, to minimize possible bias, treatment efficacy of combination treatments was assumed to be equal between ASE and OLA, and assumption was made that, during maintenance, in mixed episodes patients efficacy in relapse prevention was similar for both treatments arms.

Model robustness was tested using both deterministic and probabilistic sensitivity analyses; results proved the model reliability.

Another possible limitation could be that only EPS and weight gain has been considered among possible SGAs adverse events. Nevertheless, since ASE showed a better safety profile compared to OLA with regard to metabolic effects including dyslipidemia and type 2 diabetes mellitus [45], the impact of other adverse events could possibly generate a better performance for ASE.

\section{CONCLUSION}

Results from this study suggest that the management of bipolar I patients with mixed episodes using ASE as alternative to OLA can lead to cost savings for Italian NHS and improve patients' quality of life. Future researches specifically developed for this subset of patients are needed to fill the gap of information that are required by the costeffectiveness model and to permit the comparison of ASE with other SGAs.

\section{ACKNOWLEDGMENTS}

This study and the article processing charges were supported by an unrestricted grant from Lundbeck Italy SpA, which was not responsible 
for the study, data analysis, data interpretation, or manuscript writing. All named authors meet the ICMJE criteria for authorship for this manuscript, take responsibility for the integrity of the work as a whole, and have given final approval for the version to be published.

Conflict of interest. CC is an employee of Lundbeck S.p.A. GDS has received honoraria from CSD Medical Research Srl. AF is/has been a consultant and/or a speaker for Angelini, Astra Zeneca, Bristol-Myers Squibb, Boehringer Ingelheim, Pfizer, Eli Lilly, Jannssen, Lundbeck, Novartis, Sigma Tau, and Takeda, and has received honoraria from CSD Medical Research Srl. GM has received grant/funding from Lundbeck and has served as an advisory board from Eli Lilly, Lundbeck, Angelini, Bristol Myers Squibb, and Pfizer, and has received honoraria from CSD Medical Research Srl. GP has acted as consultant of Sanofi Aventis, Bristol Myers Squibb, Astra Zeneca, Eli Lilly, Lundbeck; received grant/research support from Eli Lilly, Astra Zeneca, Glaxo-SmithKline; is on the speaker/advisory board of Sanofi Aventis, Bristol Myers Squibb, Astra Zeneca, Eli Lilly, Jannsen-Cilag, and Lundbeck, and has received honoraria from CSD Medical Research Srl. CR is an employee of CSD Medical Research Srl. CV has received honoraria from CSD Medical Research Srl. Otherwise, the authors report no conflicts of interest in this work.

Compliance with ethics guidelines. The analysis in this article is based on previously conducted studies and does not involve any new studies of human or animal subjects performed by any of the authors.

Open Access. This article is distributed under the terms of the Creative Commons
Attribution Noncommercial License which permits any noncommercial use, distribution, and reproduction in any medium, provided the original author(s) and the source are credited.

\section{REFERENCES}

1. American Psychiatric Association, Diagnostic and Statistical Manual of Mental Disorders, 4th ed. Washington, D.C., USA, 2000.

2. Fagiolini A, Forgione R, Maccari $M$, et al. Prevalence, chronicity, burden and borders of bipolar disorder. J Affect Disord. 2013;148(2-3):161-9.

3. Murray CJ, Lopez AD. Global mortality, disability, and the contribution of risk factors: global Burden of Disease Study. Lancet. 1997;349(9063):1436-42.

4. Das Gupta R, Guest JF. Annual cost of bipolar disorder to UK society. $\mathrm{Br} \mathrm{J}$ Psychiatry. 2002;180:227-33.

5. Fountoulakis KN, Kontis D, Gonda X, Siamouli M, Yatham LN. Treatment of mixed bipolar states. Int J Neuropsychopharmacol. 2012;15(7):1015-26.

6. Zajecka JM. Calming the bipolar storm: treating acute mania and mixed episodes in patients with bipolar disorder. CNS Spectr. 2005;11(Suppl 13):1-16.

7. Perugi G, Akiskal HS, Micheli C, et al. Clinical subtypes of bipolar mixed states: validating a broader European definition in 143 cases. J Affect Disord. 1997;43(3):169-80.

8. Akiskal HS, Benazzi F. Psychopathologic correlates of suicidal ideation in major depressive outpatients: is it all due to unrecognized (bipolar) depressive mixed states? Psychopathology. 2005;38(5):273-80.

9. Akiskal HS, Benazzi F, Perugi G, Rihmer Z. Agitated, "unipolar" depression re-conceptualized as a depressive mixed state: implications for the antidepressant-suicide controversy. J Affect Disord. 2005;85(3):245-58.

10. Zarate CA, Tohen MF. Bipolar disorder and comorbid substance use disorders, in Substance abuse in the mentally and physically disabled, Chap. 4. New York: Marcel Dekker Inc; 2005. p. 64.

11. Swann AC, Lafer B, Perugi G, et al. Bipolar mixed states: an international society for bipolar disorders task force report of symptom structure, course of 
illness, and diagnosis. Am J Psychiatry. 2013;170(1):31-42.

12. Filaković P, Petek Erić A, Požgain I. New strategies in the treatment of bipolar disorder. Psychiatria Danubina. 2011;23(3):293-9.

13. Grunze H, Azorin JM. Clinical decision making in the treatment of mixed states. World $\mathrm{J}$ Biol Psychiatry. 2014 (Epub ahead of print).

14. Muralidharan K, Ali M, Silveira LE, et al. Efficacy of second generation antipsychotics in treating acute mixed episodes in bipolar disorder: a meta-analysis of placebo-controlled trials. J Affect Disord. 2013;150(2):408-14.

15. McIntyre RS, Tohen M, Berk M, Zhao J, Weiller E. DSM-5 mixed specifier for manic episodes: evaluating the effect of depressive features on severity and treatment outcome using asenapine clinical trial data. J Affect Disord. 2013;150(2):378-83.

16. Saphris: Highlights of prescribing information. Available from: https://www.merck.com/product/ usa/pi_circulars/s/saphris/saphris_pi.pdf (Last accessed April 01, 2014).

17. Azorin JM, Sapin C, Weiller E. Effect of asenapine on manic and depressive symptoms in bipolar I patients with mixed episodes: results from post hoc analyses. J Affect Disord. 2013;145(1):62-9.

18. McIntyre RS, Cohen M, Zhao J, Alphs L, Macek TA, Panagides J. A 3-week, randomized, placebocontrolled trial of asenapine in the treatment of acute mania in bipolar mania and mixed states. Bipolar Disord. 2009;11(7):673-86.

19. McIntyre RS, Cohen M, Zhao J, Alphs L, Macek TA, Panagides J. Asenapine in the treatment of acute mania in bipolar I disorder: a randomized, doubleblind, placebo-controlled trial. J Affect Disord. 2010;122(1):27-38.

20. McIntyre RS, Cohen M, Zhao J, Alphs L, Macek TA, Panagides J. Asenapine versus olanzapine in acute mania: a double-blind extension study. Bipolar Disord. 2009;11(8):815-26.

21. All Wales Medicines Strategy Group (AWMSG). Aripiprazole tablets for the treatment of moderate to severe manic episodes in Bipolar I Disorder and for the prevention of a new manic episode in patients who experienced predominantly manic episodes and whose manic episodes responded to aripiprazole. AWMSG Secretariat Assessment Report Advice, no. 1209, 2009.

22. Klok RM, Al Hadithy AF, van Schayk NP, et al. Pharmacoeconomics of quetiapine for the management of acute mania in bipolar I disorder. Expert Rev Pharmacoecon Outcomes Res. 2007;7(5):459-67.

23. McKendrick J, Cerri KH, Lloyd A, D'Ausilio A, Dando S, Chinn C. Cost effectiveness of olanzapine in prevention of affective episodes in bipolar disorder in the United Kingdom. J Psychopharmacol. 2007;21(6):588-96.

24. Ramirez de Arellano A, Rubio-Terres C, Baca E, et al. Economic evaluation analysis in the treatment of bipolar disorder with aripiprazole and other atypical antipsychotic drugs in Spain. Presented at ISPOR 13th Annual European Congress, Prague, 2010.

25. Chisholm D, van Ommeren M, Ayuso-Mateos JL, Saxena S. Cost-effectiveness of clinical interventions for reducing the global burden of bipolar disorder. $\mathrm{Br} \mathrm{J}$ Psychiatry. 2005;187: 559-67.

26. Fajutrao L, Paulsson B, Liu S, Locklear J. Costeffectiveness of quetiapine plus mood stabilizers compared with mood stabilizers alone in the maintenance therapy of bipolar I disorder: results of a Markov model analysis. Clin Ther. 2009;31(1):1456-68.

27. Sawyer L, Azorin JM, Chang S, et al. Costeffectiveness of asenapine in the treatment of bipolar I disorder patients with mixed episodes. J Med Econ. 2014;17(7):508-19.

28. Lachaine J, Beauchemin C, Mathurin K, Gilbert D, Beillat M. Cost-effectiveness of asenapine in the treatment of bipolar disorder in Canada. BMC Psychiatry. 2014;14:16.

29. Ekman M, Lindgren P, Miltenburger C, Meier G, Locklear JC, Chatterton ML. Cost effectiveness of quetiapine in patients with acute bipolar depression and in maintenance treatment after an acute depressive episode. Pharmacoeconomics. 2012;30(6):513-30.

30. Soares-Weiser K, Bravo Vergel Y, Beynon S, et al. A systematic review and economic model of the clinical effectiveness and cost-effectiveness of interventions for preventing relapse in people with bipolar disorder. 2007;11(39):iii-iv, ix-206.

31. Nivoli AM, Colom F, Murru A, et al. New treatment guidelines for acute bipolar depression: a systematic review. J Affect Disord. 2011;129(1-3):14-26.

32. Szegedi A, Calabrese JR, Stet L, et al. Asenapine as adjunctive treatment for acute mania associated with bipolar disorder: results of a 12-week core study and 40-week extension. J Clin Psychopharmacol. 2012;32(1):46-55. 
33. Vieta E, Suppes T, Ekholm B, Udd M, Gustafsson U. Long-term efficacy of quetiapine in combination with lithium or divalproex on mixed symptoms in bipolar I disorder. J Affect Disord. 2012;142(1-3):36-44.

34. Vieta E, Günther O, Locklear J, et al. Effectiveness of psychotropic medications in the maintenance phase of bipolar disorder: a meta-analysis of randomized controlled trials. Int J Neuropsychopharmacol. 2011;14(8):1029-49.

35. ISTAT. (2013) ISTAT_-Health for all. Available from: http://www.istat.it/it/archivio/14562 (Last accessed April 01, 2014).

36. Angst F, Stassen HH, Clayton PJ, Angst J. Mortality of patients with mood disorders: follow-up over 34-38 years. J Affect Disord. 2002;668(2-3):167-81.

37. Tohen M, Baker RW, Altshuler LL, et al. Olanzapine versus divalproex in the treatment of acute mania. Am J Psychiatry. 2002;159(6):1011-7.

38. Niufan G, Tohen M, Qiuqing A, et al. Olanzapine versus lithium in the acute treatment of bipolar mania: a double-blind, randomized, controlled trial. J Affect Disord. 2008;105(1-3):101-8.

39. Ara R, Brazier JE. Populating an economic model with health state utility values: moving toward better practice. Value Health. 2010;13(5):509-18.

40. CODIFA. Informatore Farmaceutico (2014). Available from: http://www.codifa.it/ (Last accessed April 01, 2014).
41. Ministero della Salute. (2014) Ministero della Salute. Available from: http://www.salute.gov.it/ (Last accessed April 01, 2014).

42. NICE. National Institute for Health and Care Excellence (2013). Available from: http:// publications.nice.org.uk/guide-to-the-methods-oftechnology-appraisal-2013-pmg9 (Last accessed April 01, 2014).

43. Brunelle J, Consoli A, Tanguy $\mathrm{ML}$, et al. Phenomenology, socio-demographic factors and outcome upon discharge of manic and mixed episodes in hospitalized adolescents: a chart review. Eur Child Adolesc Psychiatry. 2009;18(3):185-93.

44. Ouanes S, Chenoufi L, Cheour M. An update on the treatment of mixed bipolar states: what is new in 2013? J Affect Disord. 2014;158:53-5.

45. Kemp DE, Zhao J, Cazorla P, et al. Weight change and metabolic effects of asenapine in patients with schizophrenia and bipolar disorder. J Clin Psychiatry. 2014;75(3):238-45.

46. Hayhurst H, Palmer S, Abbott R, Johnson T, Scott J. Measuring health-related quality of life in bipolar disorder: relationship of the EuroQol (EQ-5D) to condition-specific measures. Qual Life Res. 2006;15(7):1271-80.

47. Briggs A, Wild $\mathrm{D}$, Lees $\mathrm{M}$, et al. Impact of schizophrenia and schizophrenia treatment-related adverse events on quality of life: direct utility elicitation. Health Qual Life Outcomes. 2008;6:105. 